\title{
More sweetness than light? A search for the causes of diabetic vasculopathy
}

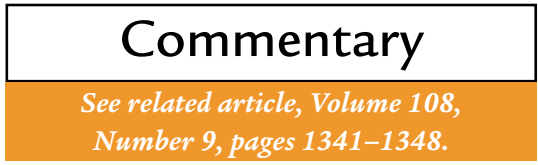

\author{
Junsuke Igarashi ${ }^{1}$ and Thomas Michel ${ }^{1,2}$ \\ ${ }^{1}$ Cardiovascular Division, Brigham and Women's Hospital, Harvard Medical School, Boston, Massachusetts, USA \\ ${ }^{2}$ Veterans Affairs Boston Healthcare System, West Roxbury, Massachusetts, USA
}

Address correspondence to: Thomas Michel, Cardiovascular Division, Brigham and Women's Hospital,

Thorn Building, Room 1210A, 75 Francis Street, Boston, Massachusetts 02115, USA.

Phone: (617) 732-7376; Fax: (617) 732-5132; E-mail: michel@calvin.bwh.harvard.edu.

J. Clin. Invest. 108:1425-1427 (2001). DOI:10.1172/JCI200114508.

Diabetes affects a large and growing number of individuals in industrialized countries worldwide; in the USA, over 16 million people suffer from diabetes, and even more show clinical features associated with prediabetic states (1). Vascular diseases represent an important cause of the morbidity and mortality associated with diabetes, and a very large body of experimental work has sought to elucidate the cellular and molecular mechanisms that underlie this important pathophysiological process. One of the hallmarks of diabetic vascular disease is endothelial dysfunction, which may be functionally defined as the failure of the vascular endothelium to subserve its normal role in vasodilation and/or vascular homeostasis.

\section{eNOS modification and the regulation of vascular tone}

Nitric oxide (2), synthesized in the vascular endothelium by the endothelial isoform of nitric oxide synthase (eNOS), represents one of the most important endothelium-derived bioactive molecules. eNOS has therefore become a focus of much investigation aimed at understanding the molecular derangements associated with diabetic vascular disease. However, despite a broad array of experimental approaches, the molecular mechanisms underlying diabetic vasculopathy remain incompletely understood, and many plausible pathophysiological models lack definitive experimental validation. Yet another model may be proposed based on a report published in a recent issue of the JCI (3), in which Du et al. present evidence that the eNOS molecule itself appears to be $O$-glyco- sylated in endothelial cells exposed to high glucose concentrations.

O-linked glycosylation has been reported to occur for a broad array of cellular proteins, including nuclear, cytoplasmic, and membrane-associated proteins (4). In contrast to the wellunderstood pathways of protein $\mathrm{N}$-glycosylation characteristic of many transmembrane proteins (which involve the $N$-linked ligation of multiple linked sugar moieties), O-glycosylation occurring in the cytoplasm typically involves the addition of a single sugar, usually $\mathrm{N}$-acetylglucosamine (abbreviated GlcNAc) to the protein's serine and threonine residues. This observation has raised the intriguing possibility that protein serine/threonine phosphorylation, a well-established pathway for enzyme regulation, might be perturbed by $O$-glycosylation (4). Although O-GlcNAcylation is proposed to reciprocally attenuate $O$-phosphorylation at specific serine/ threonine residues, the enzymatic pathways that might modulate protein $O$-glycosylation remain incompletely understood.

eNOS can be activated in complex ways, both by hemodynamic shear stress and by a variety of calciummobilizing agonists that bind to cell surface receptors in vascular endothelial cells. eNOS catalysis employs as cosubstrates molecular oxygen and NADPH, as well as a broad array of enzyme-bound redox-sensitive cofactors (including heme and tetrahydrobiopterin), to oxidize the amino acid L-arginine to form NO plus L-citrulline. Changes in cell redox state might impinge upon any of a variety of these cofactors and may also affect the bioavailability of the NO synthesized by the enzyme. eNOS itself is subject to numerous covalent modifications, including acylation by the fatty acids myristate and palmitate, and this enzyme also undergoes phosphorylation (see review in ref. 5). eNOS-activating agonists such as bradykinin, sphingosine 1-phosphate, VEGF, and insulin can differentially regulate eNOS phosphorylation through the activation of distinct protein kinase pathways (6). eNOS subcellular targeting within endothelial cells may also have important implications for diabetes-associated alterations in eNOS regulation. eNOS is targeted to specialized microdomains of plasma membrane termed caveolae, where eNOS is tonically inhibited by caveolin, a scaffolding protein of caveolae (5). Interestingly, insulin receptors may be similarly targeted to caveolae and are also regulated by caveolin (7).

The complexity of eNOS regulation and the labile nature of its product permit the coexistence of numerous pathophysiological pathways whereby diabetes might impinge upon $\mathrm{NO}$ signaling. NO signal transduction may be affected by alterations in substrate availability, by changes in eNOS expression, by derangement of the signaling pathways that connect receptor stimulation to eNOS enzyme activation (reviewed in ref. 8), by changes in cellular tetrahydrobiopterin (9) or NADPH (10) levels, or by the quenching of bioactive NO by glucose (11), lipoproteins (12), or reactive oxygen species (ROS) (8), among numerous other mechanisms. Thus, it is already possible to construct a wide array of molecular models that could explain the alterations in NO-dependent signaling seen in diabetic vasculopathy. The current 


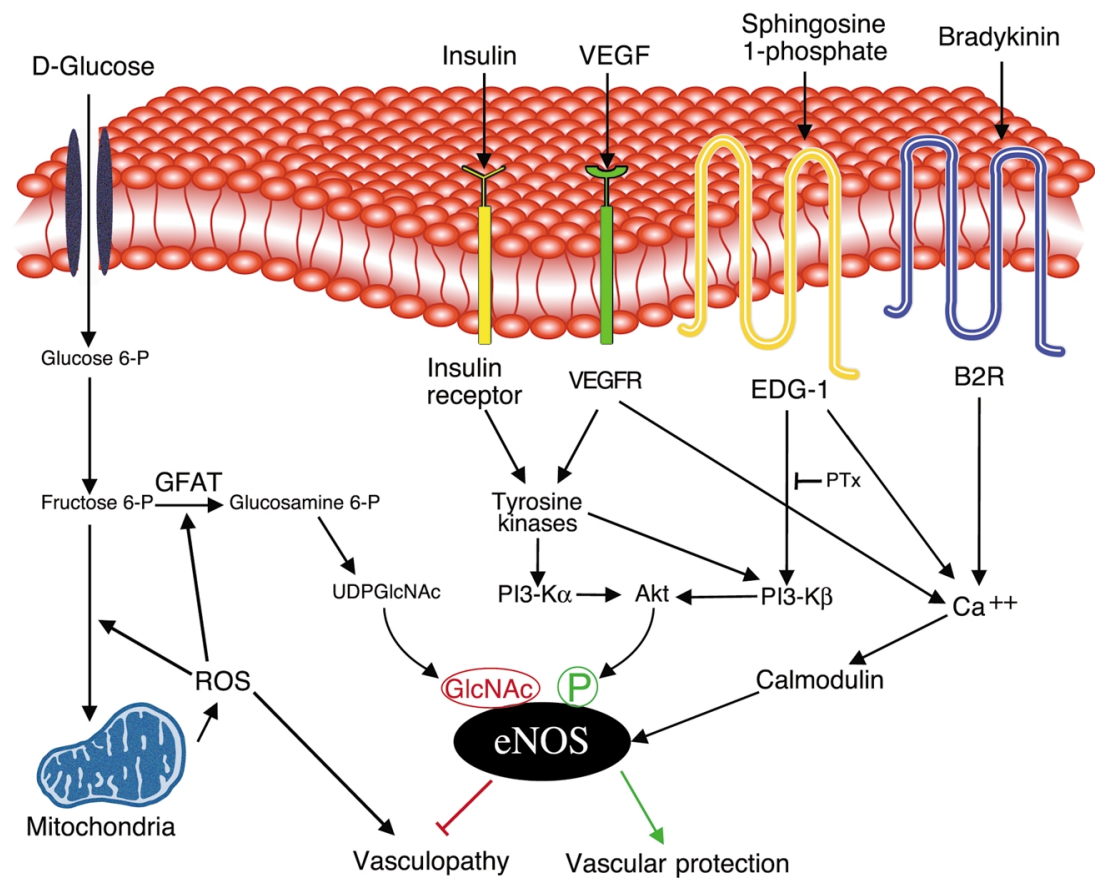

Figure 1

Signaling pathways that may mediate eNOS glycosylation and phosphorylation in vascular endothelial cells. The left half of the figure shows a model based on the results presented by Du et al. in a recent issue of the $\mathrm{JCl}(3)$. High-glucose treatment of endothelial cells is suggested to lead to the augmentation of ROS from mitochondria, leading to the activation of the glucosamine pathway by the activation of glutamine:fructose-6-phosphate amidotransferase (GFAT, the key enzyme in this pathway), ultimately increasing eNOS O-glycosylation. Basal levels of eNOS phosphorylation (green) at serine 1179 may be reciprocally attenuated by eNOS O-glycosylation with $\mathrm{N}$-acetylglucosamine (GlcNAc; red). Various eNOS agonist receptors, such as those for insulin (17), VEGF (15), and sphingosine 1-phosphate (6), are also shown (right half of the figure). These receptors typically reside in or translocate to the caveolae (shown here as a membrane invagination). Each of these cell surface receptor pathways ultimately converges at the levels of eNOS enzyme activation, but each appears to involve distinct intervening calcium-mobilizing and protein kinase pathways to activate eNOS (6). Growth factors appear to activate both the $\alpha$ and the $\beta$ isoforms of PI 3-kinase, whereas the G protein-coupled EDG-1 receptor activates only the PI 3- $\beta$ isoform. Bradykinin does not appear to activate any PI 3-kinase isoform, and its effects are mediated by pertussis toxin-insensitive $G$ proteins. By contrast, the platelet-derived agonist sphingosine 1-phosphate activates EDG-1 receptors that are coupled via pertussis toxin-sensitive $G$ proteins. The effects of diabetes on these receptor-regulated dynamic eNOS phosphorylation pathways remain to be determined. It will be interesting to explore whether the counterbalancing of eNOS O-glycosylation and phosphorylation influences the development of diabetic vasculopathy, as suggested by Du et al. (3).

suggestion that eNOS is controlled by $O$-glycosylation (3) represents the newest addition to this list of molecular mechanisms (see Figure 1) whereby diabetic conditions could affect this crucial regulator of vascular function.

\section{O-glycosylation of eNOS}

To simulate diabetic vascular conditions in vitro, Du et al. (3) incubated endothelial cells in high-glucose culture conditions for 2 days, increasing the concentration of $\mathrm{D}$-glucose in tissue culture media from $5 \mathrm{mM}$ to 30 $\mathrm{mM}$ (corresponding to glucose concentrations of 90 and $540 \mathrm{mg} / \mathrm{dl}$, respectively). The same group previ- mediated hexosamine pathway. They report here that basal NO production is significantly lower in endothelial cells cultured under high-glucose conditions, and they suggest that glucose treatment leads to a decrease in eNOS serine phosphorylation in direct parallel to the observed increase in eNOS glycosylation. Following up on this intriguing association, the authors also show increased eNOS-associated $\mathrm{O}-\mathrm{GlcNAc}$ immunoreactivity in extracts isolated from the blood vessels of diabetic rats.

How much light do these studies shed on eNOS glycosylation as a regulatory mechanism in diabetes? One important limitation of the present work relates to the in vitro model system used to provide the bulk of the data. Although this experimental approach is widely used and appears to yield reproducible data, growing cells on plastic for a few days in the presence of elevated glucose clearly represents an imperfect model for studying the complex systemic metabolic derangements characteristic of diabetes in vivo. In the in vivo experiments shown, while the apparent increase in eNOS O-glycosylation in blood vessels from diabetic rats is consistent with a pathophysiological role for this posttranslational modification, it would have been helpful to normalize the eNOS glycosylation data to the levels of other endothelial markers. Future work will also need to address the question of eNOS phosphorylation in vivo in the diabetic vasculature, which was not examined in the present report.

Even beyond these concerns, the significance of eNOS O-glycosylation remains uncertain because the specific molecular pathways and broader cellular consequences of this protein modification are still incompletely characterized. In particular, it will be important to determine the stoichiometry of this modification on eNOS. Unfortunately, the sites of eNOS glycosylation have not been not determined, and the specificity of the anti-GlcNAc and anti-phosphoserine antibody remains incompletely established in this cellular system. Moreover, there are additional sites for eNOS phosphorylation, including serine 116 (15), whose interactions with the more extensively characterized phosphorylation site at serine 1179 may be important in determining eNOS activity. 
Finally, the authors have only studied eNOS phosphorylation in the basal state, whereas it is clear that eNOS phosphorylation at serine 1179 is dynamically regulated by the kinase Akt (Figure 1), which itself is activated by phophoinositide (PI) 3-kinase isoforms that are in turn modulated by diverse extracellular stimuli, including VEGF (16); mechanical stress (17); sphingosine 1-phosphate, a plateletderived sphingolipid mediator (6); and insulin itself (18). How are these dynamic responses altered (if at all) in cells grown in the presence of elevated glucose? The relevance of such questions to diabetes is underscored by several studies that have shown that insulin stimulates eNOS phosphorylation (18), as well as by in vivo studies that document that the muscarinic agonist-modulated activation of eNOS occurs independently of blood glucose concentration (19).

Thus, the hypothesis connecting eNOS $O$-glycosylation to diabetic vasculopathy, while original and intriguing, is only incompletely tested. However, despite some experimental limitations and the broader uncertainties in the field, the novel and potential- ly important report of Du et al. (3) will undoubtedly stimulate further investigation that will shed even more light on the possible role of protein $O$-glycosylation in diabetic vascular disease.

1. McKinlay, J., and Marceau, L. 2000. US public health and the 21 st century: diabetes mellitus. Lancet. 356:757-761.

2. Loscalzo, J., and Welch, G. 1995. Nitric oxide and its role in the cardiovascular system. Prog. Cardiovasc. Dis. 38:87-104.

3. Du, X.L., et al. 2001. Hyperglycemia inhibits endothelial nitric oxide synthase activity by posttranslational modification at the Akt site. J. Clin Invest. 108:1341-1348.

4. Hart, G.W. 1997. Dynamic O-linked glycosylation of nuclear and cytoskeletal proteins. Annu. Rev. Biochem. 66:315-335.

5. Michel, T., and Feron, O. 1997. Nitric oxide synthases: which, where, how, and why? J. Clin. Invest. 100:2146-2152.

6. Igarashi, J., and Michel, T. 2001. Sphingosine 1phosphate and isoform-specific activation of phosphoinositide 3-kinase beta: evidence for divergence and convergence of receptor-regulated endothelial nitric oxide synthase signaling pathways. J. Biol. Chem. 276:36281-36288.

7. Yamamoto, M., et al. 1998. Caveolin is an activator of insulin receptor signaling. J. Biol. Chem 273:26962-26968.

8. Harrison, D.G. 1997. Cellular and molecular mechanisms of endothelial cell dysfunction. $J$ Clin. Invest. 100:2153-2157.

9. Katusic, Z.S. 2001. Vascular endothelial dysfunction: does tetrahydrobiopterin play a role? Am. J. Physiol. Heart Circ. Physiol. 281:H981-H986.

10. Asahina, T., et al. 1995. Impaired activation of glucose oxidation and NADPH supply in human endothelial cells exposed to $\mathrm{H} 2 \mathrm{O} 2$ in high-glucose medium. Diabetes. 44:520-526.

11. Brodsky, S.V., Morrishow, A.M., Dharia, N., Gross, S.S., and Goligorsky, M.S. 2001. Glucose scavenging of nitric oxide. Am. J. Physiol. Renal Physiol. 280:F480-F486.

12. Chin, J.H., Azhar, S., and Hoffman, B.B. 1992 Inactivation of endothelial derived relaxing factor by oxidized lipoproteins. J. Clin. Invest. 89:10-18.

13. Nishikawa, T., et al. 2000. Normalizing mitochondrial superoxide production blocks three pathways of hyperglycaemic damage. Nature. 404:787-790.

14. Du, X.L., et al. 2000. Hyperglycemia-induced mitochondrial superoxide overproduction activates the hexosamine pathway and induces plasminogen activator inhibitor-1 expression by increasing Sp1 glycosylation. Proc. Natl. Acad. Sci. USA. 97:12222-12226.

15. Gallis, B., et al. 1999. Identification of flow-dependent endothelial nitric-oxide synthase phosphorylation sites by mass spectrometry and regulation of phosphorylation and nitric oxide production by the phosphatidylinositol 3-kinase inhibitor LY294002. J. Biol. Chem. 274:30101-30108.

16. Fulton, D., et al. 1999. Regulation of endothelium-derived nitric oxide production by the protein kinase Akt. Nature. 399:597-601.

17. Dimmeler, S., et al. 1999. Activation of nitric oxide synthase in endothelial cells by Akt-dependent phosphorylation. Nature. 399:601-605.

18. Montagnani, M., Chen, H., Barr, V.A., and Quon, M.J. 2001. Insulin-stimulated activation of eNOS is independent of $\mathrm{Ca} 2+$ but requires phosphorylation by Akt at Ser1179. J. Biol. Chem. 276:30392-30398

19. Steinberg, H.O., et al. 1996. Obesity/insulin resistance is associated with endothelial dysfunction. Implications for the syndrome of insulin resistance. J. Clin. Invest. 97:2601-2610. 